\title{
A Bayesian estimation on right censored survival data with mixture and non-mixture cured fraction model based on beta-Weibull distribution
}

\begin{abstract}
Models for survival data that includes the proportion of individuals who are not subject to the event under study are known as a cure fraction models or simply called long-term survival models. The two most common models used to estimate the cure fraction are the mixture model and the non-mixture model. in this work, we present mixture and the non-mixture cure fraction models for survival data based on the beta-Weibull distribution. This four parameter distribution has been proposed as an alternative extension of the Weibull distribution in the analysis of lifetime data. This approach allows the inclusion of covariates in the models, where the estimation of the parameters was obtained under a Bayesian approach using Gibbs sampling methods.
\end{abstract}

Keyword: Beta-Weibull distribution; Survival data; Cure fraction 